\title{
Nanomechanical resonators operating as charge detectors in the nonlinear regime
}

\author{
H. Krömmer, A. Erbe, A. Tilke, S. Manus, and R.H. Blick* \\ Center for NanoScience and Sektion Physik, Ludwig-Maximilians-Universität, \\ Geschwister-Scholl-Platz 1, 80539 München, Germany.
}

(February 28, 2022)

\begin{abstract}
We present measurements on nanomechanical resonators machined from Silicon-on-Insulator substrates. The resonators are designed as freely suspended $\mathrm{Au} / \mathrm{Si}$ beams of lengths on the order of $1-4 \mu \mathrm{m}$ and a thickness of $200 \mathrm{~nm}$. The beams are driven into nonlinear response by an applied modulation at radio frequencies and a magnetic field in plane. The strong hysteresis of the magnetomotive response allows sensitive charge detection by varying the electrostatic potential of a gate electrode.
\end{abstract}

77.65.Fs, 73.40.Gk, 73.23.Hk

Typeset using REVTEX 
The mechanical vibration of a violin string produces audible sounds in the frequency range of some $100 \mathrm{~Hz}$ to several $10 \mathrm{kHz}$. Halving the length of such a clamped string the eigenfrequencies are increased by an octave. Scaling down the string to only some $100 \mathrm{~nm}$ yields frequencies in the radio frequency (RF) range. Recent work on such nanomechanical resonators [1,2] demonstrated different schemes of detection and their versatility for applications in metrology [3 [- Integrating mechanically flexible structures with single electron devices on the nanometer scale offers not only high speed of operation but also broad tunability of the tunnel contacts. Applications of mechanical resonators in nonlinear oscillators [6.0] or parametric amplifiers [8] are of great importance for scanning probe measurements and accurate frequency counters or clocks in general.

In this work, we want to demonstrate how to build nanometer sized mechanical resonators and how to apply the nonlinear response of these devices for charge detection at several $10 \mathrm{MHz}$. The resonators are operated in the RF regime with typical dimensions of only a few $100 \mathrm{~nm}$ in width and height. Applying a sufficiently large excitation amplitude, the suspended beam shows a highly nonlinear response, which in turn allows extremely sensitive charge detection. Moreover, the device represents a model to studying phenomena such as stochastic resonance and deterministic chaos in a mechanical system on the nanometer scale.

The material employed is a commercially available Silicon-on-insulator (SOI) substrate with thicknesses of the Si-layer and the sacrificial layer of $205 \mathrm{~nm}$ and $400 \mathrm{~nm}$, respectively. Processing of the devices requires optical lithography in a first step by which a metallic $\mathrm{Al} / \mathrm{Au}(180 \mathrm{~nm})$ mask is deposited. Adjacently the nanostructure is defined by electronbeam lithography and deposition of an $\mathrm{Al} / \mathrm{Au}$-layer with a total thickness of typically $80 \mathrm{~nm}$. The metal layers deposited on Si during lithography are a thin adhesion layer of $\mathrm{Ni} / \mathrm{Cr}(1.5 \mathrm{~nm})$, a covering Au-layer $(50 \mathrm{~nm})$, and an Al-etch mask $(30 \mathrm{~nm})$. The sample then is dry-etched in a reactive-ion etcher (RIE) in order to obtain a mesa structure with clear cut walls. Finally, we perform a hydro-fluoric wet-etch step to remove the sacrificial layer below the resonators. 
The suspended resonator is shown in Fig 1: This particular beam has a length of $l=$ $3 \mu \mathrm{m}$, a width of $w=200 \mathrm{~nm}$ and a height of $h=250 \mathrm{~nm}$ and is clamped on both sides. The gate contact couples on the complete length of the resonator. The remaining beam is a $\mathrm{Au} / \mathrm{Si}$ hybrid for which different elastic moduli have to be taken into account in the numerical simulations (not shown here). From these and the measurements shown below we can conclude that the HF attacked the Si-beam slightly, hence the resonance frequency is lower than usual. This enhances the nonlinear response of the beam, since the restoring forces are less rigid. This assumption is verified by the close-up of the suspended beam shown in the inset of Fig. 1.

All measurements were conducted at $4.2 \mathrm{~K}$ in a sample holder with a residual ${ }^{4} \mathrm{He}$-gas pressure of about $10^{-2}$ bar. This ensures thermal coupling, while it naturally reduces the mechanical quality factor (defined as $\kappa=f / \Delta f$, where $f$ is the frequency at resonance and $\Delta f$ half width of the maximum). The sample is mounted between interconnecting microstrip lines, designed to feed the circuit with frequencies up to $10 \mathrm{GHz}$, and a magnetic field is applied perpendicular to the beam. The absolute resistance of the metal wire on top of the resonator was found to be $34 \Omega$, which results in an appropriate impedance matching of the circuit. The beam is set into motion by applying a high frequency electromagnetic excitation in the range of $10-100 \mathrm{MHz}$ and ramping a magnetic field in plane. This results in an effective Lorentz force generated perpendicular to the sample surface. For excitation and final amplification we use an HP 8594A spectrum analyzer. The hysteresis of the mechanical resonator is probed with an additional Marconi 2032 source, which can be ramped from lower to higher frequencies and vice versa. The preamplifier employed is a low-noise broad-band (UHF- to L-band) JS amplifier (MITEQ Corp. 1998) with a specified noise figure of $N F=0.6 \mathrm{~dB}$ and gain of $G=30 \mathrm{~dB}$.

The capacitive coupling between beam and gate (see inset Fig. 1) is determined by numerical evaluation with a commercially available program (MAFIA, electromagnetic problem solver, ver. 3.20). ¿From these calculations we obtain a capacitive coupling between gate and beam in the linear regime of $C_{g b} \cong 220 \mathrm{aF}$. The frequency shift $\delta f$ of the mechanical 
resonance results from the capacitive coupling given by the electrostatic energy $E=Q^{2} / 2 C$, where $Q$ is the accumulated charge and $C$ the capacitance between resonator and gate. This term can be expanded with regard to the elongation amplitude $u=u(t)$ of the suspended beam, which yields for the electrostatic energy with $C=C(u)$ via a truncated Taylor expansion

$$
E(u)=\frac{1}{2} \frac{Q^{2}}{C} \cong \frac{1}{2} \frac{Q^{2}}{C+\frac{1}{2} C^{\prime \prime} u^{2}} \cong \frac{1}{2} \frac{Q^{2}}{C}\left(1-\frac{1}{2} \frac{C^{\prime \prime}}{C} u^{2}\right)=E-\frac{1}{4} \frac{Q^{2}}{C^{2}} C^{\prime \prime} u^{2},
$$

where $C^{\prime \prime}=\left.\frac{\partial^{2} C(u)}{\partial u^{2}}\right|_{u=0}$ represents the second derivative of the capacitance with respect to the spatial coordinate $u$ at $u=0$. This gives with $Q=C U$ a frequency shift of the mechanical resonance on the order of

$$
\delta f=\sqrt{f^{2}-\frac{C^{\prime \prime}}{2 m_{e f f}} V^{2}}-f \cong-\frac{C^{\prime \prime}}{4 m_{e f f} f^{2}} V^{2},
$$

where $m_{e f f}$ is the beam's effective mass (in our case $\sim 4.3 \times 10^{-16} \mathrm{~kg}$ ) and $V$ the applied gate voltage. It has to be noted that for an absolute charge measurement the necessary charging of all metallic contacts, e.g. bond pads and leads have to be taken into account. For one of the bond pads for example we estimate a capacitance of $C_{b p}=\epsilon A / d \cong 2.11 \mathrm{fF}$. However, it is still possible to determine the relative charge $\delta q$ on the closely connected gate with a high accuracy as will be shown below.

In Fig. 2(a) the RF response of the beam is depicted: Applying a magnetic field in plane, we find an increase of the peak maximum proportional to $\sim B^{2}$ (plotted in the inset). The driving amplitude of the $\mathrm{RF}$ is $-66 \mathrm{dBm}$, ensuring linear response of the suspended beam. The FWHM of the resonance is $\Delta f=(16 \pm 0.2) \mathrm{kHz}$, resulting in a mechanical quality factor at resonance of $\kappa=f_{0} / \Delta f=2330 \pm 30$. We find a large discrepancy compared to macroscopic mechanical oscillators with $\kappa \sim 10^{5}$ [6]. This can be explained by the coupling gas in the sample holder and the fact that the surface tension in these small devices naturally has a larger influence than in macroscopic systems.

In Fig. 2(b) the power coupled into the resonator is increased from $-70 \mathrm{dBm}$ to $-50 \mathrm{dBm}$ where we find a strong nonlinear response. In the present case the nonlinear response is 
identified by the distorted peak shape. Above a critical value of the excitation voltage the curve finally shows a bistability accompanied by a pronounced hysteresis. The transition occurs at about $-53 \mathrm{dBm}$, although an asymmetry of the peak structure is found at $-59 \mathrm{dBm}$ already. The nonlinearity is caused by the variation of the restoring force at the clamping points [1] and can be modelled by adding a cubic term in the equation of motion of the beam [6]. Comparing our data with a model derived earlier [7] we find excellent agreement (modelled traces are not shown).

A closer inspection of the nonlinear response seen in Fig. 2(b) can be obtained with an external frequency source, while monitoring the absorption on the spectrum analyzer. This allows to approach the hysteretic region around $f=37.287 \mathrm{MHz}$ and $37.294 \mathrm{MHz}$ from larger and lower frequencies. In Fig. 3 such a measurement is shown: The inverted triangle $(\nabla)$ corresponds to an increasing frequency, while the triangle $(\triangle)$ represents the lowering branch. The applied power is $P_{e x c}=-49 \mathrm{dBm}$ and the magnetic field $B=12 \mathrm{~T}$. Within this bistable region (width $\Delta f_{\text {hys }} \sim 7 \mathrm{kHz}$ ) the resonator is very sensitive to charge fluctuations on the nearby gate. Following this idea the suspended beam is a prime candidate to study stochastic resonance [9] in a nanomechanical resonator at RF.

Optimum operating conditions are obtained by fixing the driving amplitude at the critical point with maximum slope (traces in Fig. 4). The excitation power is levelled at $-52.8 \mathrm{dBm}$ and the magnetic field at $12 \mathrm{~T}$. As seen in the inset the peak position varies as the square of the gate voltage applied. We achieve a sensitivity of $\Delta V / \sqrt{\Delta f} \cong 4.1 \times 10^{-2} \mathrm{~V} / \sqrt{\mathrm{Hz}}$. The slope at the critical point $d A /\left.d f\right|_{f=f_{c}} \rightarrow \infty$ diverges, resulting in extremely sensitive amplification. In the measurements presented in Fig. 4 we obtain a charge resolution at a finite bias on the gate $(V= \pm 4 \mathrm{~V})$ of $\sim 0.7 \times 10^{2} \mathrm{e} / \sqrt{\mathrm{Hz}}$ limited by electronic noise. It is important to note the enhancement of sensitivity with increasing gate voltage (see inset of Fig. 4). The accuracy of the measurement can be further enhanced by determining the phase shift of the resonator, as shown in Fig. 5. For this measurement we modified our arrangement according to Ref. [3], i.e. including a mixer and a phase shifter. With this setup it was possible to obtain a sensitivity of $\sim 1.0 \times 10^{-1} \mathrm{e} / \sqrt{\mathrm{Hz}}$. As before the operating 
point is adjusted in the transistion region at the critical point (as indicated in Fig. 5). Biasing the resonator to $V= \pm 4 \mathrm{~V}$, we obtain a charge resolution of $\delta q=n e=70 e$.

In summary, we demonstrated a new method to apply nanomechanical resonators as charge detection devices at radio frequencies. The main features are the high speed of operation and the increased sensitivity, due to the operation in the nonlinear regime. An interesting outlook is given by considering the quantum limit for the resonators applied in this work: Assuming a bath temperature of $T_{b}=10 \mathrm{mK}$ and computing the relation $k_{B} T_{b} / h=f_{m e c h}$, we obtain a frequency limit of about $200 \mathrm{MHz}$ for the quantum mechanical oscillation regime (with $k_{B}$ : Boltzmann constant; $h$ : Planck's constant).

We like to thank Jörg P. Kotthaus for his support and for helpful discussions. Also we thank Klaus Richter and Richard J. Warburton for critically reading the manuscript. This work was funded in part by the Bundesministerium für Forschung und Technologie (BMBF) and the Deutsche Forschungsgemeinschaft (DFG).

*: Corresponding author: Robert H. Blick / Electronic mail: robert.blick@physik.unimuenchen.de 


\section{REFERENCES}

[1] A.N. Cleland and M.L. Roukes, Appl. Phys. Lett. 69, 2653 (1996).

[2] D.W. Carr, L. Sekaric, and H.G. Craighead, J. Vac. Sci. Technol. B 16, 3821 (1998); D. W. Carr and H. G. Craighead, J. Vac. Sci. Technol. B 15, 2760 (1997).

[3] A.N. Cleland and M.L. Roukes, Nature 392, 160 (1998).

[4] A. Erbe, R.H. Blick, A. Tilke, A. Kriele, J.P. Kotthaus, Appl. Phys. Lett. 73, 3751 (1998).

[5] Ch. Weiss and W. Zwerger, Europhys. Lett. 47, 97 (1999).

[6] D.S. Greywall, B. Yurke, P.A. Busch, A.N. Pargellis, and R.L. Willett, Phys. Rev. Lett. 72, 2992 (1994);

[7] B. Yurke, D.S. Greywall, A.N. Paragellis, P.A. Busch, Phys. Rev. A 51, 4211 (1995).

[8] D. Rugar and P. Grütter, Phys. Rev. Lett. 67, 699 (1991).

[9] L. Gammaitoni, F. Marechesoni, E. Menichella-Saetta, and S. Santucci, Phys. Rev. Lett. 62, 349 (1989); L. Gammaitoni, P. Hänggi, P. Jung, F. Marchesoni, Rev. Mod. Phys. 70, $223(1998)$. 


\section{FIGURES}

Fig. 1: Micrograph of the nanomechanical resonator used in the experiment: The gate couples to the resonator, machined out of $\mathrm{Si}$ with a $50 \mathrm{~nm}$ evaporated Au-layer. Inset: Close-up of the resonator applied in the measurements.

Fig. 2: (a) Mechanical resonance at $f=37.26 \mathrm{MHz}$ : Excitation level is fixed at $-66 \mathrm{dBm}$ with peak maximum increasing as $B^{2}$ (see inset). (b) Beam response driven into the nonlinear regime at $B=12 \mathrm{~T}$ with power levels increasing as indicated from -70 to $-50 \mathrm{dBm}$.

Fig. 3: Hysteretic response of the excited beam: The inverted triangle $(\nabla)$ indicates the signal with rising frequency, while the triangle $(\triangle)$ represents lowering of the frequency $\left(P_{e x c}=-49 \mathrm{dBm}\right.$ and $\left.B=12 \mathrm{~T}\right)$.

Fig. 4: Operating the resonator in the transition region at $-52.8 \mathrm{dBm}$ with maximum signal sensitivity. Resonance traces are shifted by an applied gate voltage. Note the shifting of the critical point when the gate voltage is varied (inset).

Fig. 5: Measured phase variation of the resonator in the transition region as in Fig. 2(b). Inset: Phase of the resonator at a fixed gate voltage. 


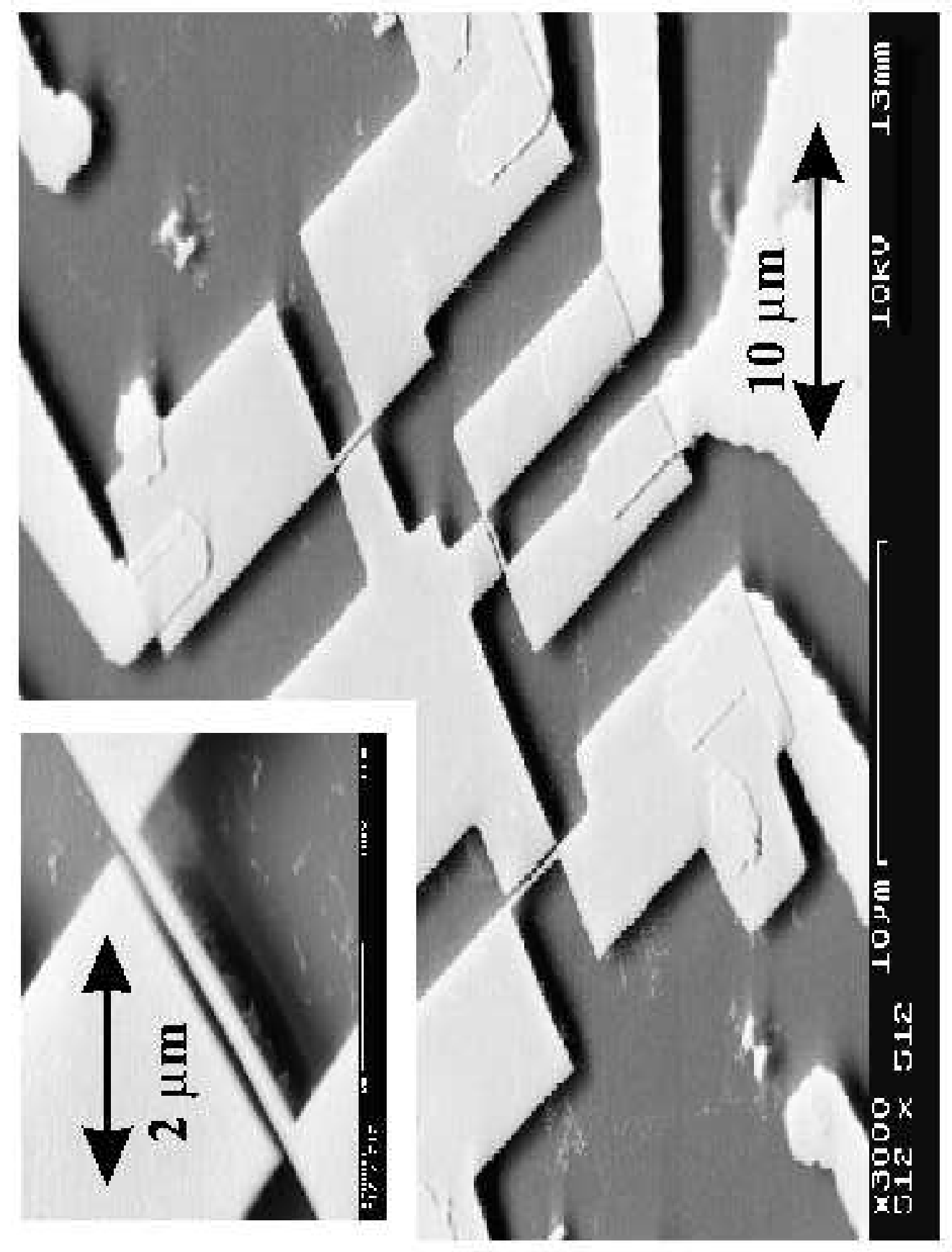




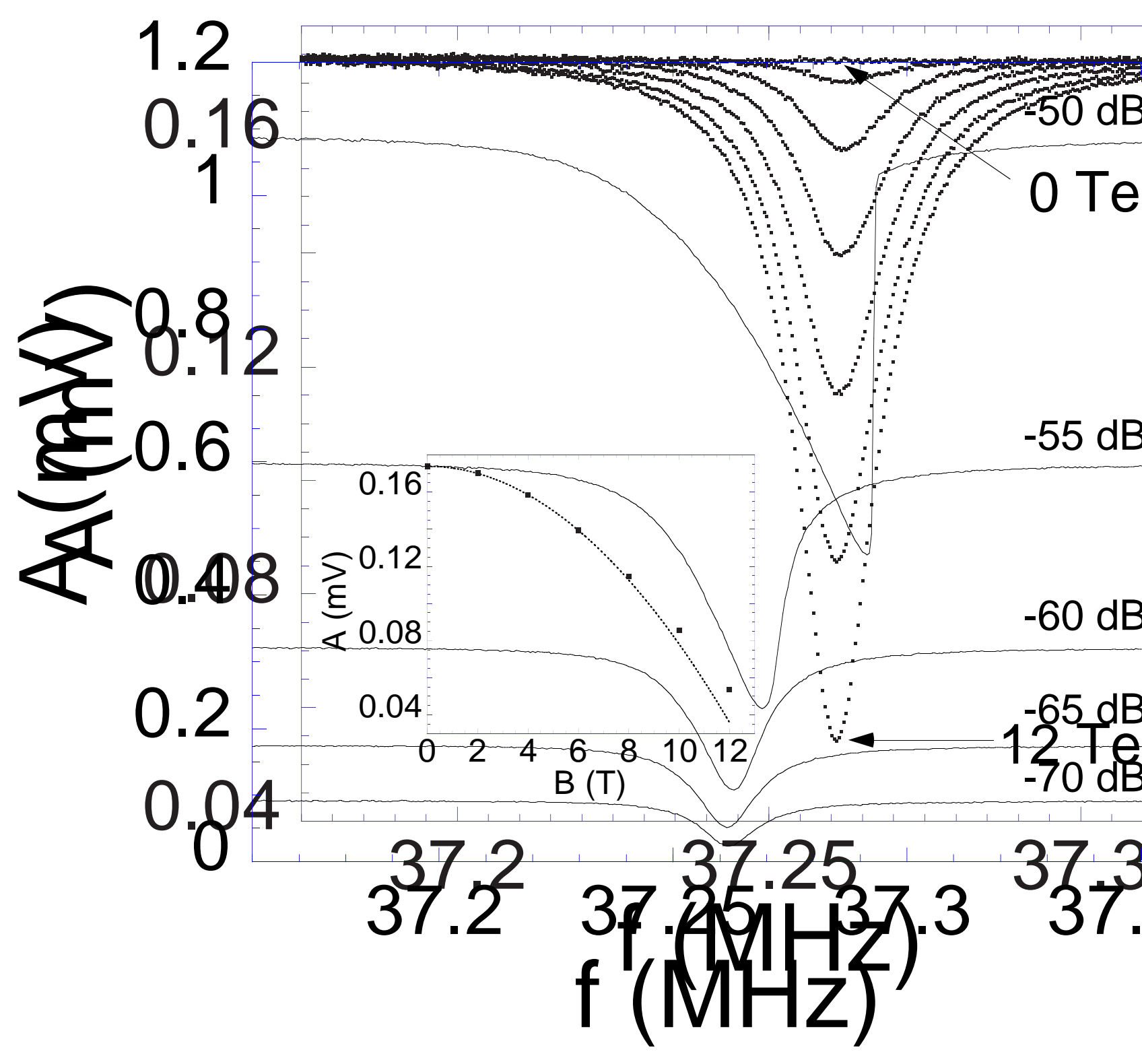




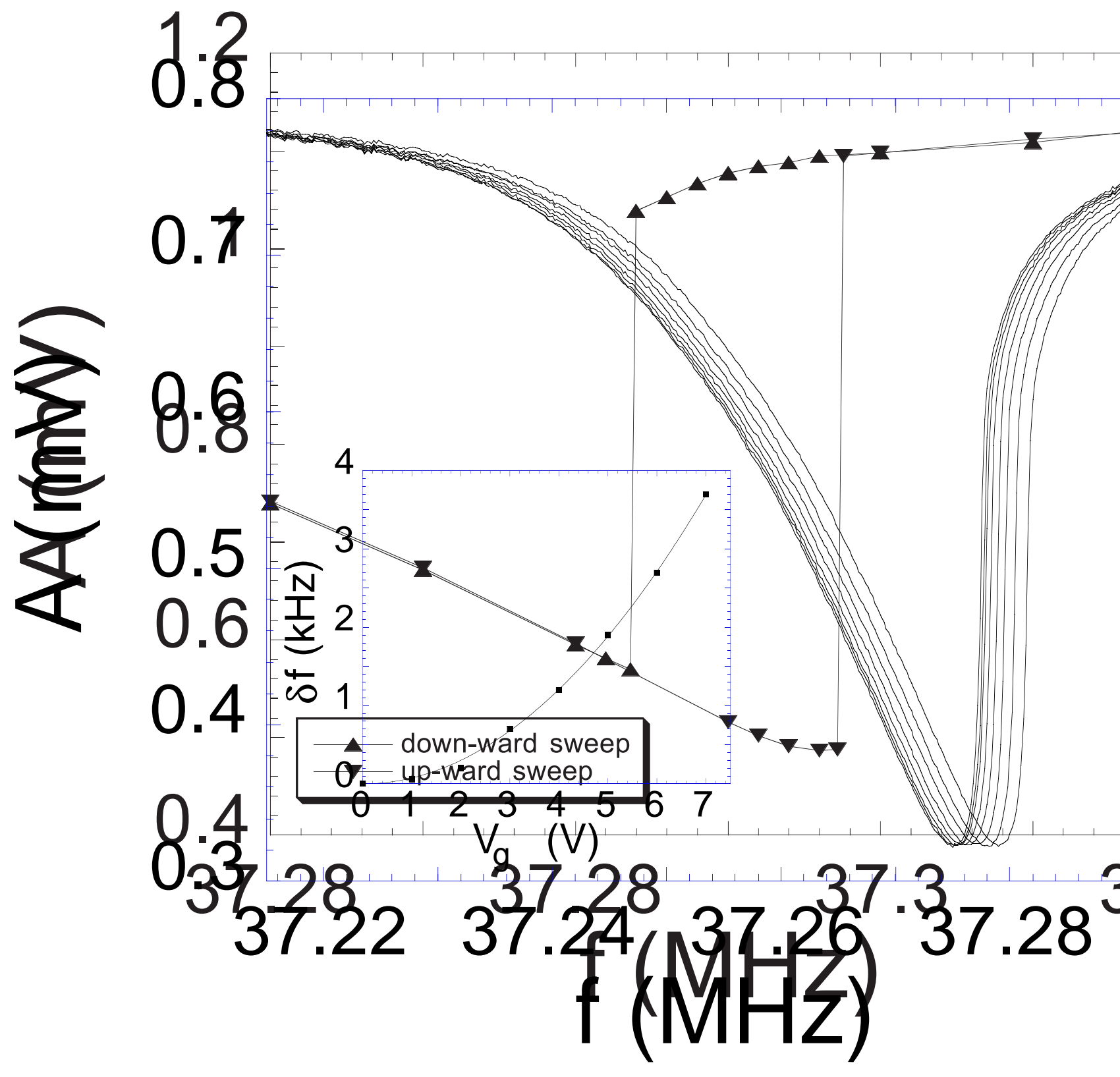




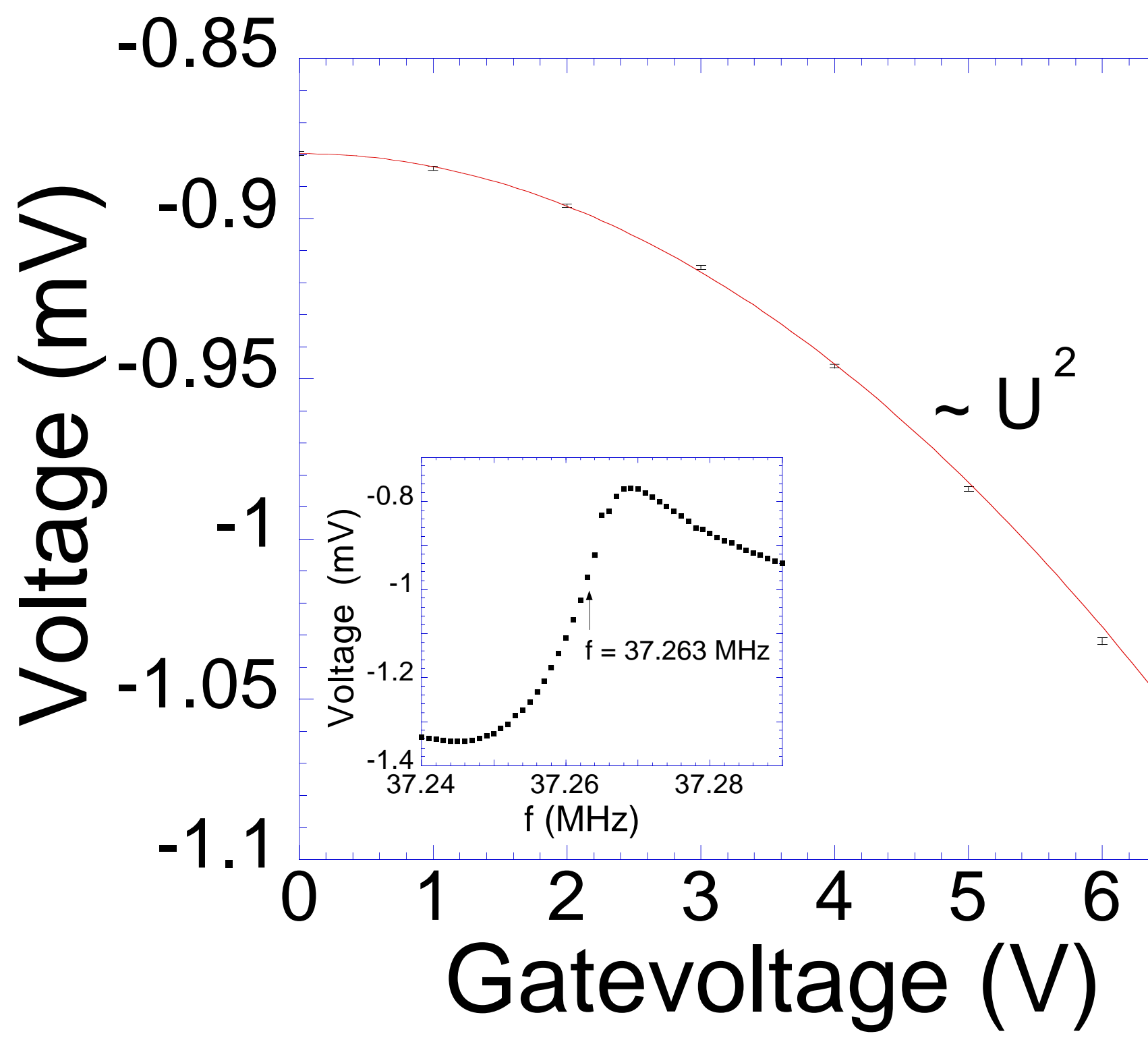

